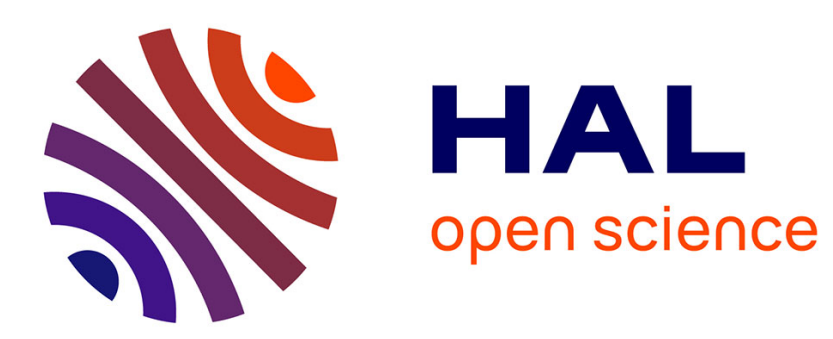

\title{
Light scattering by fractal aggregates : a Monte-Carlo method in the geometrical optics limit
}

\author{
R. Jullien, R. Botet
}

\section{To cite this version:}

R. Jullien, R. Botet. Light scattering by fractal aggregates : a Monte-Carlo method in the geometrical optics limit. Journal de Physique, 1989, 50 (14), pp.1983-1994. 10.1051/jphys:0198900500140198300 . jpa-00211042

\section{HAL Id: jpa-00211042 https://hal.science/jpa-00211042}

Submitted on 1 Jan 1989

HAL is a multi-disciplinary open access archive for the deposit and dissemination of scientific research documents, whether they are published or not. The documents may come from teaching and research institutions in France or abroad, or from public or private research centers.
L'archive ouverte pluridisciplinaire HAL, est destinée au dépôt et à la diffusion de documents scientifiques de niveau recherche, publiés ou non, émanant des établissements d'enseignement et de recherche français ou étrangers, des laboratoires publics ou privés. 
Classification

Physics Abstracts

$78.65 \mathrm{~S}-81.35 \mathrm{~T}-42.20 \mathrm{Y}$

\title{
Light scattering by fractal aggregates : a Monte-Carlo method in the geometrical optics limit
}

\author{
R. Jullien and R. Botet \\ Laboratoire de Physique des Solides, Bât. 510, Université de Paris-Sud, Centre d'Orsay, \\ 91405 Orsay, France
}

(Reçu le 14 février 1989, révisé le 4 avril 1989, accepté le 11 avril 1989)

\begin{abstract}
Résumé. - On présente une méthode Monte-Carlo pour calculer la diffusion de lumière polarisée par un ensemble de sphères identiques dans la limite de l'optique géométrique où le rayon des sphères est plus grand que la longueur d'onde du faisceau incident. Dans cette limite, on ne tient pas compte des effets de cohérence ainsi que de la diffraction. Les rayons lumineux (direction et intensité) sont suivis tout au long de leurs réflexion/réfraction en utilisant les lois de SnellDescartes et les formules de Fresnel. Sur chaque dioptre, la réflexion, ou la réfraction, est choisie au hasard et on tient compte de ce choix pour corriger l'intensité résultante. La méthode est appliquée à des agrégats fractals déterministes et désordonnés de dimensions fractales $D$ différentes, contenant jusqu'à 28561 (déterministes) et 4096 (désordonnés) sphères. La section efficace de diffusion ainsi que les intensités relatives, pour différents angles de diffusion et différentes polarisations, sont systématiquement étudiées en fonction de la taille des agrégats et des lois d'échelle simples sont obtenues. La différence de comportement entre les agrégats transparent $(D<2)$ et opaque $(D>2)$ est soulignée.
\end{abstract}

\begin{abstract}
A numerical Monte-Carlo method is presented to study the scattering of polarized light by an assembly of identical spheres in the geometrical optics limit where the radius of the spheres is larger than the wavelength of the incident beam. In this limit both coherence effects and diffraction phenomena are not taken into account. The rays (direction and intensity) are followed during their refractions/reflections using the Snell-Descartes laws and Fresnel formulae. On each diopter, refraction, or reflection, is chosen at random and the resulting scattered intensity is corrected accordingly. The method is applied to both deterministic and random fractal aggregates of various fractal dimensions $D$ containing up to 28561 (deterministic) and 4096 (random) spheres. The scattering cross-section as well as the relative intensities for various scattered angles and polarizations are systematically studied as a function of the size of the aggregates and simple scaling relations are obtained. The difference of behavior between transparent $(D<2)$ and opaque $(D>2)$ aggregates is stressed.
\end{abstract}

\section{Introduction.}

It is of great importance to study the scattering of light by an assembly of identical particles, such as aggregated colloids or aerosols, which have been recently recognized as being fractal structures [1]. A potential application can be the detection of dust or smoke. If the exact 
theory of light scattering is well known for a single sphere (the Lorentz-Mie theory [2]), there exists no exact theory for more than two spheres. Many approximate treatments have been proposed. The best known (independently of the nature of the incident wave) is the treatment which considers that all particles are ponctual and independent. The scattered intensity is then simply the square of the Fourier transform of the particle positions. This is the base of the analysis of the structure from small angle scattering experiments with $\mathrm{X}$ rays or neutrons [3]. In the case of fractal structures, this gives rise to the now famous $q^{-D}$ law [4] where $q$ is the modulus of the scattered wavevector (related to the scattered angle $\theta$ through the formula $q=(4 \pi / \lambda) \sin (\theta / 2))$ and $D$ the fractal dimension. This is the theoretical background of one of the best experimental methods to determine fractal dimensions of mass fractals.

But, it must be emphasized that this classical approach neglects two important effects :

- multiple scattering: such effect occurs when one particle is affected by the light scattered by the others. In the case of fractal aggregates, it has been recognized that multiple scattering cannot be avoided for fractal dimensions larger than two, when the aggregate becomes opaque for the incident beam [5] ;

- proximity or shadowing effects which occurs when the size of the particles cannot be neglected and when some particles can be close to each other. In principle, such effect cannot be avoided in the case of aerosol or colloid aggregates where particles are stuck together.

We have recently presented a method [6] (referenced in the following by FPBJ) able to treat multiple scattering effects inside an aggregate made of identical spherical particles, but which is limited to the Rayleigh approximation where the particle radius is smaller than the wavelength of the light, i.e. in the limit of small values of the product $k a$ where $k=2 \pi / \lambda$ is the modulus of the wavevector of the incident beam and $a$ the radius of the spheres. We are presently extending this method to all $k a$, by making use of the full LorentzMie theory [7] for the light scattered by each sphere. However, in this previous calculation, as well as in its extension, the proximity effects are not taken into account. It must be noticed that attempts have been made to treat approximately proximity effects by introducing anisotropic polarization tensors for the spheres to take care of their local environment [8].

In this paper we present a method entirely built using the geometrical optics approximation. One inconvenient compared with other methods (and in particular compared to FPBJ) is that, in this limit, only addition of incoherent waves (i.e. addition of their intensities and not of their amplitudes) have a physical meaning. Thus coherence effects cannot be recovered. In particular, one cannot find the $q^{-D}$ law. Another inconvenient is that only transmitted and reflected rays are considered, i.e. diffracted light is not considered. In practice, our method can apply to aggregates made of spherical particles whose radius is larger than the wavelength of the light (i.e. for large values of the product $k a$ ), under the condition that the angular dependence of the diffracted light can be neglected. Some of our results could eventually be extended to small $k a$ values, if there exist some physical reasons to kill coherence effects. On the other hand, the present approach has the considerable advantage to fully take into account the two effects mentioned above, i.e. multiple scattering and shadowing effects.

\section{Description of the method.}

2.1 GENERALITIES : PRODUCTION AND DETECTION OF RAYS. - The method consists in generating (far from the aggregate) a set of incident rays parallel to a given direction, defined by a unit vector $\mathbf{u}_{0}$, a given electric field $\mathbf{E}_{0}$ (whose modulus is conventionally taken equal to unity), perpendicular to $\mathbf{u}_{0}$, and issued from points $A_{0}$ regularly spaced on a square lattice of parameter $\delta$, in a plane perpendicular to $\mathbf{u}_{0}$. These rays are followed all along their trajectory inside the aggregate, and the intensity (i.e. the square of the modulus of the electric field) is 
collected at the end, for different relative polarization directions of the incident and scattered electrical field, as a function of $\theta, \theta$ being the angle between the scattered direction $\mathbf{u}_{\mathrm{s}}$ and the incident one, $\mathbf{u}_{0}$. In practice all intensities scattered between $\theta$ and $\theta+$ $\mathrm{d} \theta$ are stored in «detectors", regularly spaced in $\cos \theta$ (to obtain a quantity directly proportional to $I(\theta)$, which is usually defined by the fact that $I(\theta) \sin \theta \mathrm{d} \theta$ is the intensity scattered between $\theta$ and $\theta+\mathrm{d} \theta)$. After each reflection/refraction, here labelled by the index $i$, the impact point $\mathbf{A}_{i}$, the new direction $\mathbf{u}_{i}$ and the new electrical field $\mathbf{E}_{i}$ are exactly determined, using Snell-Descartes laws and Fresnel formulae. However, on diopter $i$, instead of collecting both rays (reflected and refracted) issued from a given incident one, a reflection or a refraction is selected at random, using a Monte-Carlo method explained below, and the collected scattered intensity is modified accordingly. Let us now develop in more details what is effectively done.

2.2 Storage of THE Aggregate. - To optimize the search for a contacted sphere, when a ray is sent at the beginning or when it escapes from another sphere, one builts, before all, a cubic lattice of parameter slightly lower than $2 a / 3^{1 / 2}$, where $a$ is the radius of the spheres, in order that no more than one sphere can be centered in each unit cube of this lattice. The minimum-sized parallelepipedic box containing the whole aggregate is determined. Let us call $x_{\mathrm{m}}, y_{\mathrm{m}}$ and $z_{\mathrm{m}}$ the lengths of its edges (in unit of the lattice spacing). One stores a $x_{\mathrm{m}}, y_{\mathrm{m}}, z_{\mathrm{m}}$ integer array, function of the integer coordinates of a cube of the lattice, equal to the number of the sphere if a sphere is centered in the cube or equal to zero in the opposite case. On the other hand one knows the list of the coordinates of the centers of the spheres as a function of their number. Then the search for a new impact point and for the corresponding sphere proceeds by following the ray and performing a systematic investigation of all the cubes located in its sufficiently nearest neighborhood to be able to eventually contain the center of a contacted sphere. After knowing the contacted sphere, one can determine all the characteristics of the reflection/refraction process and in particular the incident angle, $i$ and the refraction angle, $r$, for the first refraction. When an outside-inside refraction, or an internal reflection is selected, the new impact point, $A_{i+1}$ is determined as the function of the old one $\mathrm{A}_{i}$, knowing the direction $\mathbf{u}_{i}$ of the internal ray by:

$$
\mathbf{O A} \mathbf{A}_{i+1}=\mathbf{O A}_{i}+2 a \cos r \mathbf{u}_{i}
$$

where $O$ is the center of the sphere.

2.3 DETERMINATION OF THE NEW RAY AFTER REFLECTION/REFRACTION. - Knowing the relative refractive index $n$ of the considered sphere (compared with the outside medium), its center $\mathrm{O}$, the considered impact point $\mathrm{A}_{i}$, the old ray direction $\mathbf{u}_{i-1}$, as well as the angles $i$ and $r$, calculated for the first contact with the sphere, the new direction $\mathbf{u}_{i}$ is given by the Snell-Descartes laws which can be written here under the following forms, according to the chosen process :

- external reflection :

$$
\mathbf{u}_{i}=\mathbf{u}_{i-1}+2 \cos i \mathbf{O A} \mathbf{A}_{i} / a
$$

- outside-inside refraction :

$$
\mathbf{u}_{i}=\mathbf{u}_{i-1} / n+(\cos i-n \cos r) \mathbf{O A}_{i} /(n a)
$$

— internal reflection :

$$
\mathbf{u}_{i}=\mathbf{u}_{i-1}-2 \cos r \mathbf{O A} \mathbf{A}_{i} / a
$$


— inside-outside refraction :

$$
\mathbf{u}_{i}=n \mathbf{u}_{i-1}+(\cos i-n \cos r) \mathbf{O} \mathbf{A}_{i} / a .
$$

To determine the new field $\mathbf{E}_{i}$, one determines the projections of the old one $\mathbf{E}_{i-1}$ perpendicular and parallel to the incidence plane (defined by $\mathbf{u}_{i-1}$ and $\mathbf{O A}_{i}$ ), $E_{i-1, \text { per }}$ and $E_{i-1, \text { par }}$. One then calculates $E_{i \text {, per }}$ and $E_{i \text {, par }}$ by multiplying the old components by the reflection coefficients $r_{\mathrm{par}}$ and $r_{\mathrm{per}}$, or by the refraction coefficients $t_{\mathrm{par}}$ and $t_{\text {per }}$. The new field is then built by addition of its components (taking care of the new direction). These coefficients are given by the Fresnel formulae :

- external reflection :

$$
\begin{aligned}
& r_{\mathrm{par}}=-(\cos r-n \cos i) /(\cos r+n \cos i) \\
& r_{\mathrm{per}}=(\cos i-n \cos r) /(\cos i+n \cos r)
\end{aligned}
$$

- outside-inside refraction :

$$
\begin{aligned}
& t_{\mathrm{par}}=2 \cos i /(\cos r+n \cos i) \\
& t_{\mathrm{per}}=2 \cos i /(\cos i+n \cos r)
\end{aligned}
$$

— internal reflection :

$$
\begin{aligned}
& r_{\mathrm{par}}=(\cos r-n \cos i) /(\cos r+n \cos i) \\
& r_{\mathrm{per}}=-(\cos i-n \cos r) /(\cos i+n \cos r)
\end{aligned}
$$

— inside-outside refraction :

$$
\begin{aligned}
& t_{\mathrm{par}}=2 n \cos r /(\cos r+n \cos i) \\
& t_{\mathrm{per}}=2 n \cos r /(\cos i+n \cos r) .
\end{aligned}
$$

2.4 PRINCIPLES OF THE MONTE-CARLO METHOD. - When a ray contacts the $i$-th diopter, one generates a random number uniformly distributed between 0 and 1 . If this number is lower than a given number $p_{i}$, a reflection is chosen and, in the opposite case, a refraction is chosen. One then collects a number $q_{i}$ which is equal to $p_{i}$, if a reflection is chosen, and equal to $1-p_{i}$, if a refraction is chosen. At the end, the intensity collected in the detector $\theta$ is divided by the cumulative product of the $q_{i}$ 's : $Q=\prod q_{i}$. One can easily be convinced that this procedure gives the exact result in the limit of an infinite number of incident rays. Suppose that $N$ rays ( $N$ large) are sent with the same $\mathrm{A}_{0}$ and $\mathbf{u}_{0}$, one then will collect only $N Q$ rays in the scattered direction $\theta$, thus one must divide the collected quantities (here the intensities) by $Q$ to respect the exact proportion of one scattered ray in this direction $\theta$ for one incident ray. We have checked that the results do not depend on the choice of the $p_{i}$ 's. To uniformize the relative errors in the different scattered directions and to obtain a realistic visualization of the simulation we have chosen $p_{i}$ equal to the mean reflection coefficient for the intensities, i.e. $p_{i}=(1 / 2)\left(r_{\text {per }}^{2}+r_{\text {par }}^{2}\right)$, where $r_{\text {per }}$ and $r_{\text {par }}$ are the reflection coefficients for the two polarizations of the field at the considered $i$ diopter.

2.5 DETERMINATION OF THE INTENSITIES FOR THE DIFFERENT DIRECTIONS OF THE POLARISER AND THE ANALYSER. - The interesting quantities to be stored in the detectors are the intensities $I_{\mathrm{VV}}, I_{\mathrm{HH}}, I_{\mathrm{VH}}, I_{\mathrm{HV}}$, where the first index refers to the polarization of the incident ray and the second one to the polarization of the scattered ray, $\mathrm{V}$ denotes a polarization 
perpendicular to the scattered plane and $\mathrm{H}$, a polarization parallel to this plane. The scattered plane, which is parallel to both $\mathbf{u}_{0}$ and $\mathbf{u}_{\mathrm{s}}$, is only defined at the end, when the ray is collected in the detector. Then the projections of both $\mathbf{E}_{0}$ and $\mathbf{E}_{\mathrm{s}}$, perpendicular and parallel to the scattered plane, are determined. One knows that, in general these projections are related by :

$$
\begin{aligned}
& E_{\mathrm{s}, \mathrm{per}}=a_{\mathrm{VV}} E_{0, \mathrm{per}}+a_{\mathrm{HV}} E_{0, \mathrm{par}} \\
& E_{\mathrm{s}, \mathrm{par}}=a_{\mathrm{VH}} E_{0, \mathrm{per}}+a_{\mathrm{HH}} E_{0, \mathrm{par}}
\end{aligned}
$$

$I_{\mathrm{VV}}, I_{\mathrm{HV}}, I_{\mathrm{VH}}$ and $I_{\mathrm{HH}}$ are the squares of the matrix elements $a_{\mathrm{VV}}, a_{\mathrm{HV}}, a_{\mathrm{VH}}$ and $a_{\mathrm{HH}}$. To calculate these four quantities, it is necessary to send twice a ray, with the same $\mathrm{A}_{0}$ and $\mathbf{u}_{0}$, but with two different polarizations (in practice they are taken perpendicular to each other), to obtain four linear equations, which are then solved.

2.6 SUMMARY OF INPUT AND OUTPUT. - In addition to the relative optical index $n$, the radius of the spheres, $a$, and the list of the coordinates for the sphere centers, the other input parameters of our program are now defined.

- One is the spacing $\delta$ between the different rays sent with the same incident direction $\mathbf{u}_{0}$, i.e., since we use a square lattice in the incident plane wave (perpendicular to $\mathbf{u}_{0}$ ), there is one incident ray per area $\delta^{2}$. In practice, our input parameter is a number, $n_{\delta}$, and one calculates $\delta$ by $\delta=(1 / 3)\left(x_{\mathrm{m}}+y_{\mathrm{m}}+z_{\mathrm{m}}\right) / n_{\delta}$, where $x_{\mathrm{m}}, y_{\mathrm{m}}$ and $z_{\mathrm{m}}$ are defined in $\S 2.2$. The effective number of rays sent in the $\mathbf{u}_{0}$ direction is thus proportional to $n_{\delta}^{2}$.

- When one wants to make an average over different (uniformly distributed) incident directions (which corresponds to an average over all orientations of the aggregate), one defines another input number $n_{0}$ and one varies the parameters $\phi$ and $\cos \zeta$, determining $\mathbf{u}_{0}\left(u_{0 x}=\sin \zeta \cos \phi, u_{0 y}=\sin \zeta \sin \phi, u_{0 z}=\cos \zeta\right)$ by regular intervals of width $2 \pi / n_{0}$ and $2 / n_{0}$, between 0 and $2 \pi$, and between -1 and +1 , respectively. The number of orientations is then $n_{0}^{2}$.

- Another parameter $n_{\mathrm{d}}$ is the number of detectors. For each detector, $\cos \theta$ varies between -1 and +1 by steps of width $2 / n_{\mathrm{d}}$.

- We have introduced another parameter $n_{\text {rep }}$, which enters only to improve the statistics : the same incident ray (with its two different polarizations) is sent $n_{\text {rep }}$ times.

- Finally it must be noticed that an absorption coefficient can be introduced very easily. However, for simplicity, this has not been done here.

As output not only one collects the intensities for the different angles and polarizations but also the scattering cross-section, which is the integral of the intensity over all scattered angles, and which can be calculated by:

$$
\sigma=N_{\mathrm{d}} \delta^{2} /\left(\pi a^{2}\right)
$$

where $N_{\mathrm{d}}$ is the total number of rays effectively detected (i.e. having hit at least one sphere) for a given incident direction. The cross-section is here normalized, i.e. has been divided by the projected area of a sphere, to be equal to one for a single sphere.

2.7 TEST OF THE PROGRAM IN THE CASE OF A SINGLE SPHERE. - Our program has been first tested with a single sphere of refractive index $n=1.33$. In figure $1 \mathrm{a}$, we have reported the decimal logarithms of $I_{\mathrm{VV}}$ and $I_{\mathrm{HH}}$ as a function of $\theta$, obtained with the parameters $n_{\delta}=64, n_{0}=8, n_{\mathrm{d}}=64, n_{\mathrm{rep}}=16$. We recover that $I_{\mathrm{VH}}$ and $I_{\mathrm{HV}}$ are equal to zero as well known for a unique sphere. These curves are compared with a numerical calculation based on the exact formulation in the geometrical optics limit, taking care of up to 20 possible internal reflexions (Fig. 1b). When comparing the figures one can observe that all the features of the 

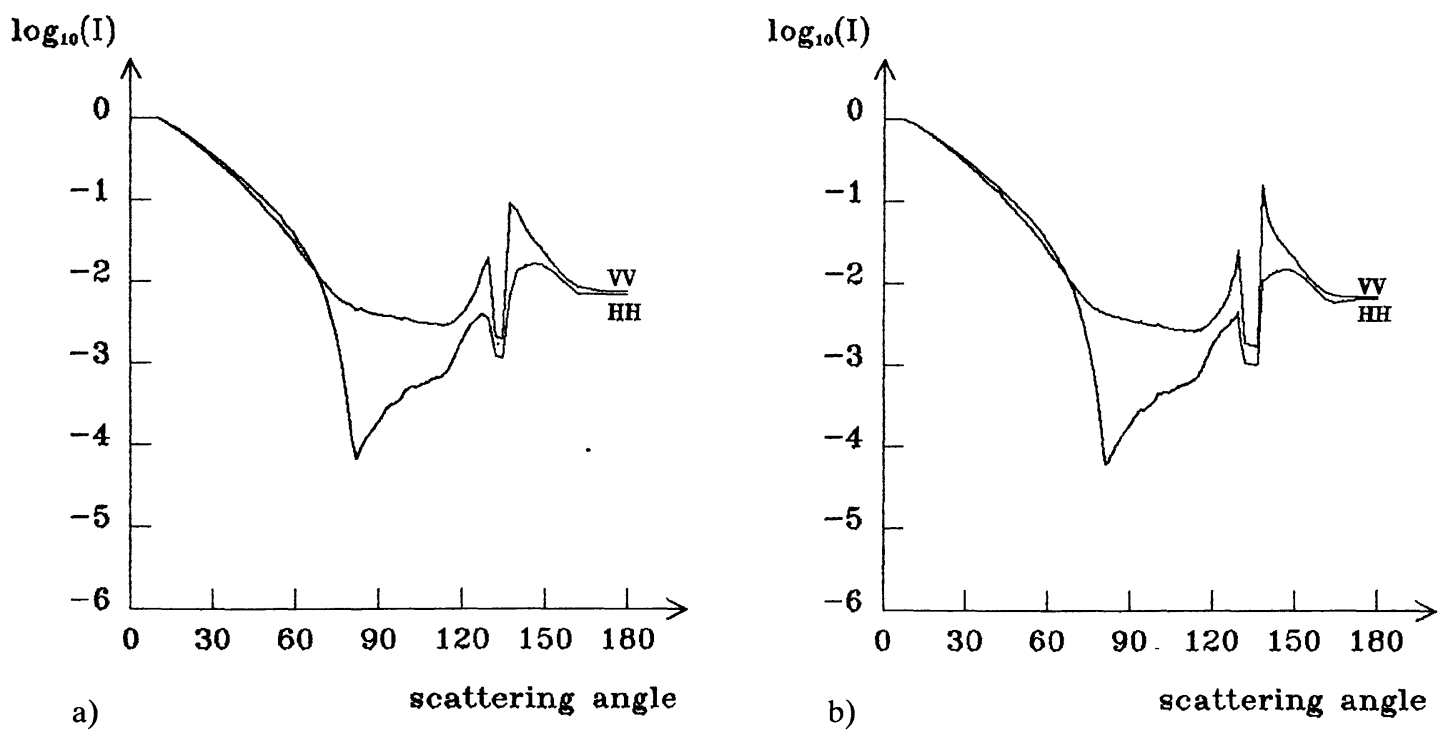

Fig. 1. - a) Decimal logarithm of the intensity of light with polarization parallel $\left(I_{\mathrm{HH}}\right)$ and perpendicular $\left(I_{\mathrm{VV}}\right)$ to the scattering plane, as a function of the scattering angle, for a single sphere, calculated with the Monte-Carlo method, using parameters $n=1.33, n_{\delta}=64, n_{0}=8, n_{\mathrm{d}}=64$, $n_{\text {rep }}=16$. b) Same quantities, calculated numerically using the exact formulae in the geometrical optics formalism, and considering up to twenty possible internal reflections for the rays issued from the same incident one.

expected curves are quite well reproduced with our method, in particular one recovers the peaks (at 130 and $138^{\circ}$ ) corresponding to the well-known « rainbow angles » [9]. The divergences are only a bit smoothed but this is not too important, especially when one knows that these divergences are artifacts due to the use of geometrical optics. We have also tested that, in that case, the reduced cross-section is equal to one with a good accuracy.

\section{Application to fractal aggregates.}

3.1 Deterministic FRACTAL AgGregAtes. - We have first considered several « deterministic " fractals built in three dimensions with an algorithm similar to the one already used by Vicsek in two dimensions [10]. One starts from a sphere centered on the origin. One then sticks a given number $n$ of other spheres on it, in directions $\mathbf{v}_{i}(i=1,2, \ldots, n)$, uniformly distributed in space. This process is repeted iteratively : at step $j, n$ aggregates identical to the central one are stuck on it in directions $\mathbf{v}_{i}$ and thus centered at points located at distance $3^{j}$ sphere diameters from the origin. At each step the mass is multiplied by $n+1$ and the diameter by 3 , so that the fractal dimension is equal to $\log (n+1) / \log (3)$. We have considered $n=6\left(\mathbf{v}_{i}=( \pm 1,0,0)\right.$ and circular permutations $), n=8\left(\mathbf{v}_{i}=\left( \pm 3^{-1 / 2}, \pm 3^{-1 / 2}\right.\right.$, $\left.\left.\pm 3^{-1 / 2}\right)\right)$ and $n=12\left(\mathbf{v}_{i}=0, \pm 2^{-1 / 2}, \pm 2^{-1 / 2}\right)$ and circular permutations $)$ corresponding to $D=1.77 \ldots, D=2$ and $D=2.33 \ldots$, respectively. In each case we have considered four generations, i.e. aggregates containing up to 2401, 6561 and 28561 spheres, respectively. To complete this series of deterministic aggregates with compact aggregates $(D=3)$, we have also considered an ensemble of cubes of increasing edges, containing tangent spheres (up to $24 \times 24 \times 24)$ regularly disposed on a simple cubic lattice. 
3.2 RANDOM FRACTAL AGGREGATES. - We have also considered several « random » fractal aggregates built by diffusion limited aggregation [11] algorithms in three dimensions and variants [12]. As in FPBJ, we have used the three-dimensional particle-cluster and clustercluster tip-to-tip models [13] leading to fractal dimensions $D=1$ and $D=1.4$, respectively, as well as the off-lattice ballistic cluster-cluster model [14], whose fractal dimension is $D=1.9$. We have also considered three-dimensional on lattice Witten-Sander aggregates whose fractal dimension is known to be $D=2.5$ [15]. In each case we have considered clusters containing $N=16,32,64, \ldots, 2048$ particles. Since we have considered only a single cluster for each calculation, and averaged over many directions of the incident beam, instead of averaging over different (statistically independent) samples, we have, before all, calculated the apparent fractal dimension of our clusters from the log-log plot of their radius of gyration versus their number of particles. We have found $D=1.1,1.4,1.9,2.4$, with an error bar of order 0.08 . To complete the set with a compact random aggregate, we have used a simple procedure [16] in which spheres are added at random to the aggregate under the conditions that they must be tangent to three other spheres (except for the second sphere which must be tangent to the first one and the third sphere which must be tangent to the two first ones) and that their distance to the first sphere must be minimum.

3.3 SUMMARY OF THE CALCULATIONS PERFORMED. - The advantage of performing calculations with deterministic fractals is that we could reach larger sizes for the simple reason that such structures can be more easily put in a small paralellepipedic box, with much less cost of memory occupancy. However, since they are anisotropic, one needs to perform a better average over the incident directions. While in the case of random fractals we were systematically running the program for numbers of particles $N$ varying as powers of 2 , in the case of deterministic fractals we were naturally limited to the four sizes of the four first generations, to avoid oscillations with the logarithm of $N$. In the case of random fractals we were using the parameters $n=1.33, n_{\delta}=128, n_{0}=8, n_{\mathrm{d}}=64, n_{\text {rep }}=4$. In the case of deterministic fractals we were using the same parameters except $n_{0}=16$ (multiplying by four the number of directions). For the largest size reached $(N=28561)$ the program was running five hours on an IBM 390.

3.4 ANGLE DEPENDENCE OF THE SCATTERED INTENSITIES. - In figure 2, we provide some typical $I(\theta)$ curves. The intensities are normalized such that $I_{\mathrm{VV}}$ and $I_{\mathrm{HH}}$ are equal to 1 for $\theta=0$. To see the effect of varying the fractal dimension we have chosen to present the curves obtained with compact random aggregates and with aggregates of very low fractal dimensions ( $D=1.1$ ) for the same numbers of particles, here $N=16$ and $N=256$. The rainbow peaks, which are always visible, are attenuated when considering larger aggregates. As a general result we find that $I_{\mathrm{VH}}$ is almost equal to $I_{\mathrm{HV}}$ : this is consistent with the fact that the scattering matrix should be symmetric for a medium which is, in average, rotationally invariant (the demonstration can be found in Bohren's book [2], pp. 412-413). We have always observed that the relative importance of $I_{\mathrm{VH}}$ and $I_{\mathrm{HV}}$ compared to $I_{\mathrm{VV}}$ and $I_{\mathrm{HH}}$ is mainly fixed by the local compactness of the aggregate : a variation of half a decade for this ratio at $\theta=0$ can be seen on the figure when going from $D=1.1$ to $D=3$ for $N=16$. Its variation with size is however characteristic of the fractal dimension. One can see on the figure that the effect of size is quite negligeable for $D=1.1$ while it is significative for the compact aggregate : not only the forward depolarization increases but also the forward to backward relative intensity decreases when the size increases. We have not studied in detail the influence of varying the refractive index $n$. While the locations of both the rainbow angles and the minimum of $I_{\mathrm{HH}}$ strongly depend on $n$, we found that the variations with size of the mean and relative intensities were quite independent of this parameter, as long as it is not taken too 


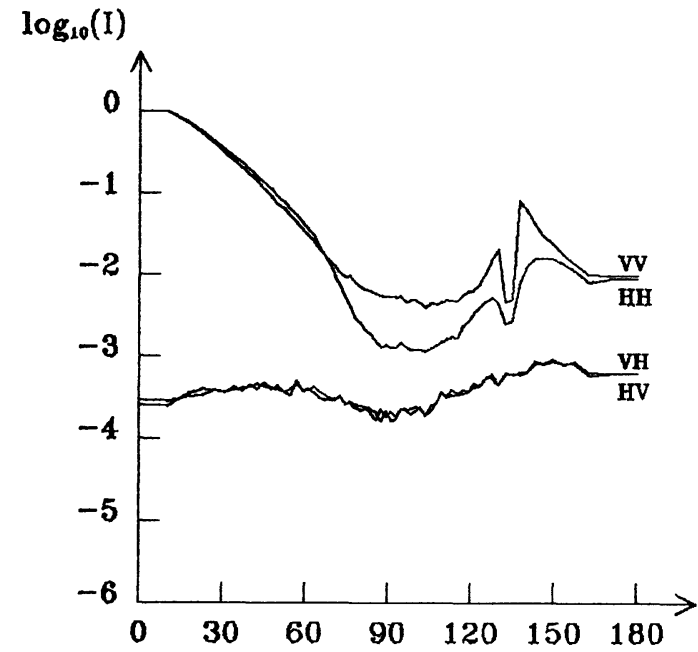

a)

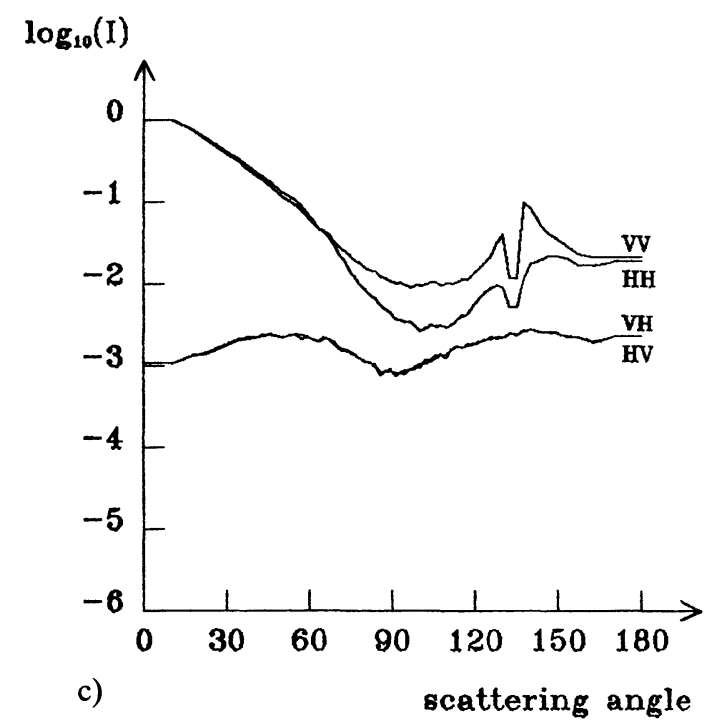

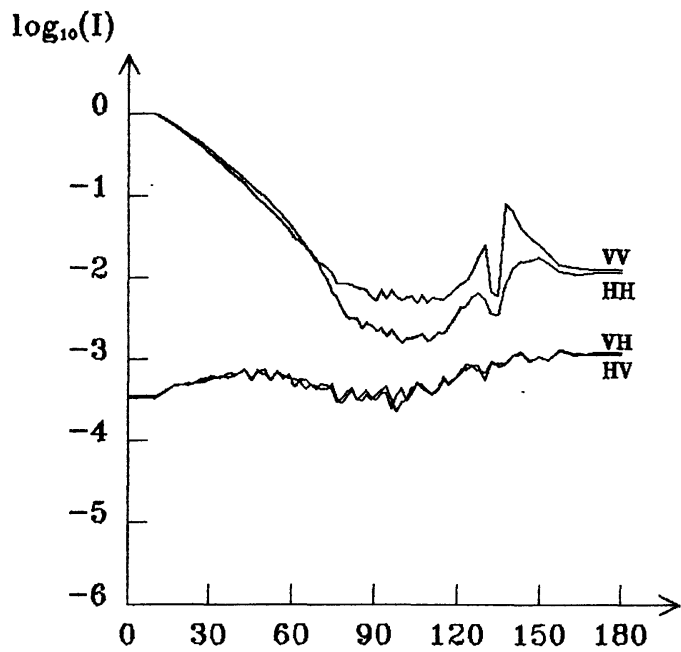

b)

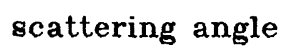

$\log _{10}(\mathrm{I})$

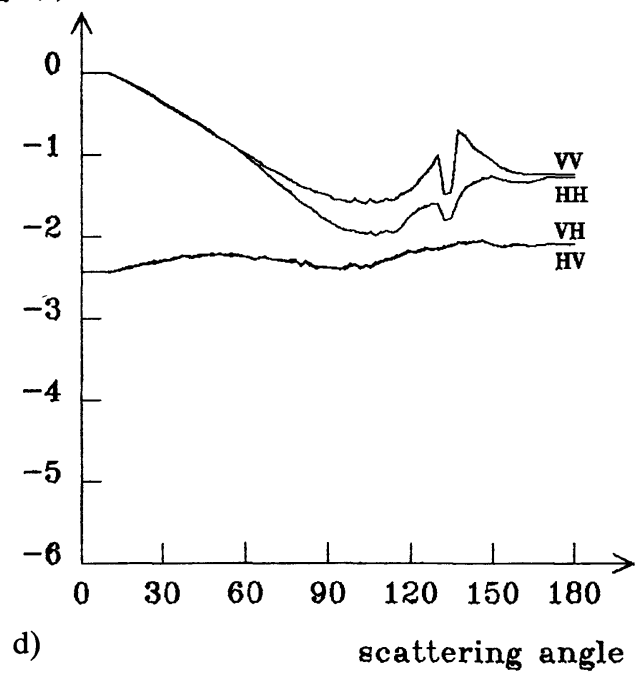

Fig. 2. - Decimal logarithm of the intensities, $I_{\mathrm{VV}}, I_{\mathrm{HH}}, I_{\mathrm{VH}}$ and $I_{\mathrm{HV}}$, as a function of the scattering angle, for fractal random aggregates of fractal dimension $D=1.1$, containing $N=16$ (a) and $N=256$ (b) spheres and for compact random aggregates $(D=3)$ containing $N=16$ (c) and $N=256$ (d) spheres. The parameters used in the calculation are $n=1.33, n_{\delta}=128, n_{0}=8$, $n_{\mathrm{d}}=64, n_{\text {rep }}=4$.

large. The case of very large $n$ corresponds to another regime (metallic spheres), not studied here, where external reflections become dominant.

3.5 CROSS-SECTION. - The cross-section, $\sigma$, as defined in section 2.6 , is proportional to the total scattered intensity integrated over all scattered directions (this is due to the fact that we have not considered any absorption here). We give the results as a log-log plot of $\sigma$ as a 
function of $N$, number of particles in the aggregate, in figures 3a (deterministic aggregates) and $3 \mathrm{~b}$ (random aggregates). Our results are consistent with $\sigma$ proportional to $N$ for $D<2$ and $\sigma$ varying as $N^{2 / D}$ for $D>2$.

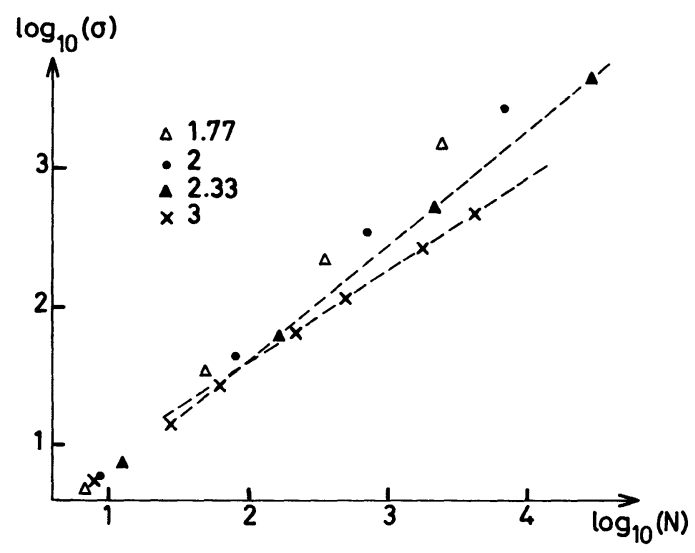

a)

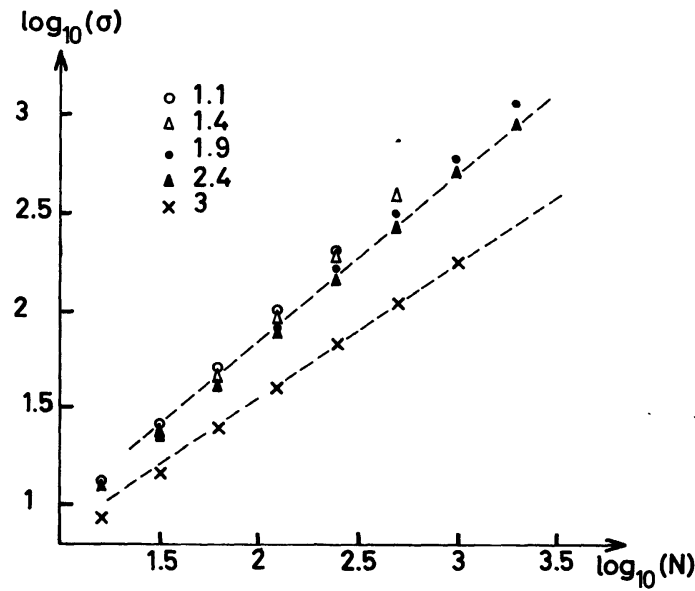

b)

Fig. 3. - Plot of the scattering cross-section $\sigma$ as a function of the number of spheres $N$ in the aggregate for deterministic (a) and for random (b) fractal aggregates (log-log plot). In the case of deterministic fractals the symbols $(\Delta, \bullet, \Delta, \times)$, correspond to fractal dimensions $D=1.77,2,2.33$ and 3 , respectively. In the case of random fractals the symbols $(0, \Delta, \bullet, \boldsymbol{\Delta}, \times)$, correspond to $D=1.1,1.4,1.9,2.4$ and 3 , respectively. For $D>2$, a straight line of slope $2 / D$ has been drawn through the data (this is not a least square fit). The same parameters as in figure 2 are used for the calculations except $n_{0}=16$ for deterministic fractals.

This is the result expected from a very simple argument : when the aggregate is transparent $(D<2)$, shadowing effects are negligible and almost all its particles are exposed to the incident light, while when it is opaque $(D>2)$, only a number of particles proportional to the square of the radius (number of particles seen on a projected area) is exposed to the incident light. It can be noticed that there might be some logarithmic corrections near $D=2$, where a slope slightly smaller than one is recovered. The same general conclusions were already reached by Paul Meakin et al. [17], who recently calculated the number of collisions between fractal aggregates and point masses moving along straight line trajectories.

It must be noticed that such shadowing effects occurring for $D>2$ could not be recovered with the previous calculations such as the one of FPBJ : there a linear dependence of the intensities with $N$ was recovered for all angles, except for $\theta=0$, where coherence effects (not taken into account here) led to a $N^{2}$ dependence.

3.6 Depolarization. - The depolarization of the scattered light can be quantitatively estimated by the relative importance of the cross intensities $I_{\mathrm{VH}}$ and $I_{\mathrm{HV}}$ compared to $I_{\mathrm{VV}}$ and $I_{\mathrm{HH}}$. We have calculated $I_{\mathrm{Vv}} / I_{\mathrm{VH}}$ for forward $(\theta=0)$ as well as for backward $\left(\theta=180^{\circ}\right)$ scattering. For the backward scattering, it is not necessary to present the results in a figure because $I_{\mathrm{VV}} / I_{\mathrm{VH}}$ is almost constant (extremely slightly decreasing) with increasing sizes and no significant change of behavior is observed around $D=2$.

For the forward scattering the results are reported in figures $4 \mathrm{a}$ (deterministic fractals) and $4 \mathrm{~b}$ (random fractals). One again clearly sees, on these curves, a change of behavior for 


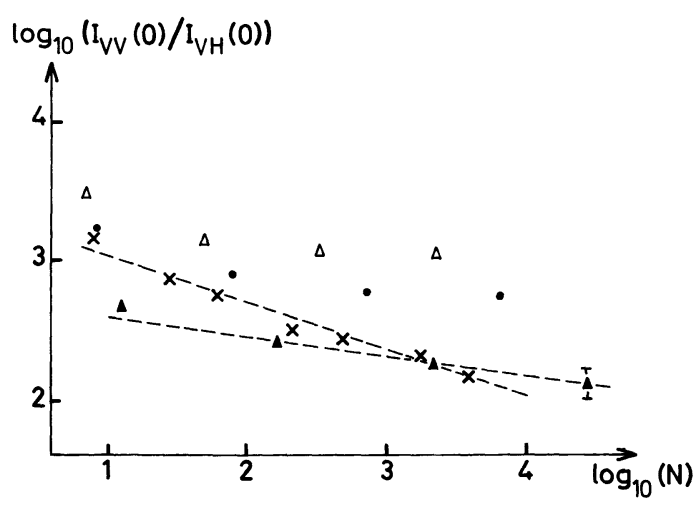

a)

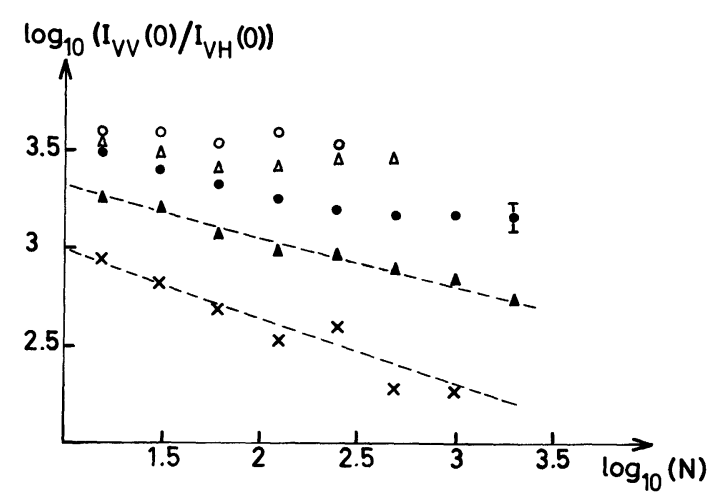

b)

Fig. 4. - Plot of $I_{\mathrm{VV}}(0) / I_{\mathrm{VH}}(0)$ as a function of the number of spheres $N$ in the aggregate for deterministic (a) and for random (b) fractal aggregates (log-log plot). For $D>2$, a straight line of slope $-(D-2) / D$ has been drawn through the data (this is not a least square fit). Same parameters and same symbols as in figure 3.

$D=2$. While $I_{\mathrm{Vv}} / I_{\mathrm{VH}}$ does not vary too much with size for $D<2$, it decreases appreciably when increasing $N$ for $D>2$. If one assumes a linear behavior for $D>2$, the estimated slope seems to be very close to $-(D-2) / D$ (again there is some ambiguity for fractals with fractal dimension close to two). This result is consistent with the fact that, as long as $I_{\mathrm{VH}}$ remains small compared to $I_{\mathrm{VV}}$, the ratio $I_{\mathrm{VH}} / I_{\mathrm{VV}}$ would be proportional to the mean number of spheres crossed by a ray passing through the aggregate. Note that this scaling cannot be valid up to $N$ infinite, since $I_{\mathrm{VH}}$ would become of the same order of magnitude as $I_{\mathrm{Vv}}$. A better quantity to study should then be $\left(I_{\mathrm{Vv}} / I_{\mathrm{VH}}-1\right)$, however the differences with $I_{\mathrm{VV}} / I_{\mathrm{VH}}$ are negligible for the sizes reached here.

It must be noticed that, while the result obtained here for backward depolarization is quite the same as that found in FPBJ, the result obtained for forward depolarization is completely different. When coherence effects are taken into account $I_{\mathrm{Vv}}$ increases much more with size (as $N^{2}$ in FPBJ where shadowing effects were neglected) and $I_{\mathrm{Vv}} / I_{\mathrm{VH}}$ is increasing instead of decreasing. It must be noticed that for large aggregates, when the central peak is very narrow, it is enough to have a very small deviation from $\theta=0$ to recover the incoherent behavior. Thus when trying to apply these results to experiments, it is essential to know whether coherence effects exist or not in the experimental intensities.

3.7 FORWARD TO BACKWARD RELATIVE INTENSITIES. - To study quantitatively the deformation of the $I(\theta)$ curves with size we have calculated the ratio between forward and backward scattering intensities $I_{\mathrm{VV}}(0) / I_{\mathrm{VV}}\left(180^{\circ}\right)$ (note that $I_{\mathrm{VV}}=I_{\mathrm{HH}}$ for $\theta=0$ and $180^{\circ}$ ). This quantity is reported in a log-log plot as a function of $N$ in figure 5 a (deterministic fractals) and $5 \mathrm{~b}$ (random fractals). The similitude with figure 4 is striking. While this quantity does not vary too much with size for $D<2$, it decreases appreciably when increasing $N$ for $D>2$ (note again the problem for fractals with fractal dimension close to two). It seems that we recover the same kind of scaling law as above : i.e. $I_{\mathrm{VV}}(0) / I_{\mathrm{Vv}}\left(180^{\circ}\right)$ scales very well like $N^{-(D-2) / D}$ for $D>2$, suggesting that this quantity is also proportional to the mean number of spheres crossed by a ray passing through the aggregate. Note that the same restriction as above should exist for very large $N$ values. When trying to apply such results to experiments, 


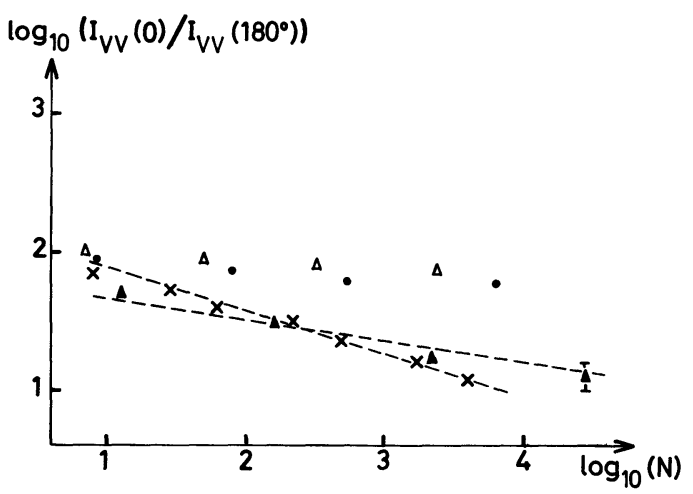

a)

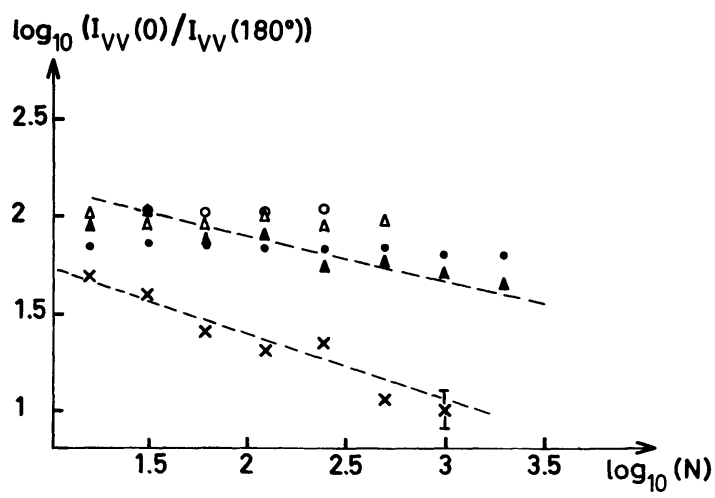

b)

Fig. 5. - Plot of $I_{\mathrm{VV}}(0) / I_{\mathrm{VV}}(180)$ as a function of the number of spheres $N$ in the aggregate for deterministic (a) and for random (b) fractal aggregates (log-log plot). For $D>2$, a straight line of slope $-(D-2) / D$ has been drawn through the data (this is not a least square fit). Same parameters and same symbols as in figure 3.

it must be remembered that the coherence peaks must be subtracted from the experimental data. This is crucial here, since $I_{\mathrm{VV}}(0)$ is concerned.

\section{Conclusion.}

We have presented a very simple minded Monte-Carlo method able to calculate the intensity of polarized light scattered by an assembly of identical spheres. This method is restricted to the geometrical optics limit where the radius of the spheres is much larger than the wavelength of light and where coherence effects as well as diffraction phenomena are not considered. The method has been applied to fractal aggregates containing up to several thousands of particles and some general scaling properties have been found: while the scattering cross-section is proportional to the number of particles and the overall shape of the $I(\theta)$ curve is quite independent of the number of particles for transparent fractal aggregates $(D<2)$, the cross-section varies like $N^{2 / D}$ and both $I_{\mathrm{VV}}(0) / I_{\mathrm{VV}}\left(180^{\circ}\right)$ and $I_{\mathrm{VV}}(0) / I_{\mathrm{VH}}(0)$ scale like $N^{-(D-2) / D}$ for opaque aggregates $(D>2)$. The generality of the results gives some hope on the domain of applicability of this method. However, when applied to aerosol and colloidal aggregates, it must be understood that coherence effects cannot be recovered. This method is complementary of the one of FPBJ which was taking care of coherence effects but was restricted to small particle sizes and was neglecting shadowing effects. We will complete this study in the near future by including absorption and by investigating the limit of large refractive indices. We will also study applications of this simple method to the scattering of light by a smog of water droplets, an application for which it is particularly well appropriate.

\section{Acknowledgments.}

We would like to acknowledge very fruitful discussions with P. Adam and J. M. Flesselles. Numerical calculations have been performed at CIRCE (Centre Inter-Régional de Calcul Electronique), Orsay, and supported by the contract DRET $n^{\circ} 87 / 1354$. 


\section{References}

[1] Forrest S. and Witten T. A., J. Phys. A 12 (1979) L109.

WeITZ D., Lin M. and Sandroff C., Surf. Sci. 158 (1985) 147.

[2] VAN De Hulst H. C., Light Scattering by Small Particles (Wiley, New York) 1957.

Bohren C. F. and HufFMAn D. R., Absorption and Scattering of Light by Small Particles (Wiley, New York) 1983.

[3] Guinier A. and Fournet G., Small Angle Scattering of X-rays (Wiley, New York) 1955.

[4] Schaeffer D., Martin J., Wiltzius P. and Cannell D., Phys. Rev. Lett. 52 (1984) 2371.

Chen S. H. and Teixeira J., Phys. Rev. Lett. 57 (1986) 2583.

Axelos M., Tchoubar D., Bottero J. and Fiessinger F., J. Phys. France 46 (1985) 1587.

[5] Berry M. and Percival I., Opt. Acta 33 (1986) 577.

[6] Frey J., Pinvidic J. J., Botet R. and Jullien R., J. Phys. France 49 (1988) 1969.

[7] BOTET R. and JULLIEN R., in preparation.

[8] Zhe Chen, Ping Sheng, Weitz D. A., Lindsay H. M., Lin M. Y. and Meakin P., AIP Conf. Proc. USA 160 (1987) 455.

[9] Nussenzveig H. M., Sci. Am. 236 (1977) 116 and references therein.

[10] VicseK T., J. Phys. A 16 (1983) L647.

[11] Witten T. A. and Sander L., M., Phys. Rev. Lett. 47 (1981) 1400.

[12] Jullien R. and BoteT R., Aggregation and Fractal Aggregates (World Scientific, Singapore) 1987.

[13] Jullien R., Phys. Rev. Lett. 55 (1985) 1697 and J. Phys. A 19 (1986) 2129.

[14] BAll R. and Jullien R., J. Phys. Lett. France 45 (1984) L1031.

[15] Bennet C. H., J. Appl. Phys. 43 (1972) 2727.

[16] Meakin P., Donn B. and Mulholland G. W., to appear in Langmuir (1989). 